\title{
Preclinical efficacy of ribavirin in SHH and group 3 medulloblastoma
}

\author{
*Sakibul Huq, BS, Nivedha V. Kannapadi, BA, Joshua Casaos, MD, Tarik Lott, BS, \\ Raphael Felder, BS, Riccardo Serra, MD, Noah L. Gorelick, MD, Miguel A. Ruiz-Cardozo, BS, \\ Andy S. Ding, BA, Arba Cecia, BS, Ravi Medikonda, BA, Jeff Ehresman, BS, Henry Brem, MD, \\ Nicolas Skuli, PhD, and Betty M. Tyler, BA
}

Hunterian Neurosurgical Research Laboratory, Department of Neurosurgery, Johns Hopkins University School of Medicine, Baltimore, Maryland

OBJECTIVE Medulloblastoma, the most common pediatric brain malignancy, has Sonic Hedgehog (SHH) and group 3 (Myc driven) subtypes that are associated with the activity of eukaryotic initiation factor 4E (elF4E), a critical mediator of translation, and enhancer of zeste homolog 2 (EZH2), a histone methyltransferase and master regulator of transcription. Recent drug repurposing efforts in multiple solid and hematologic malignancies have demonstrated that elF4E and EZH2 are both pharmacologically inhibited by the FDA-approved antiviral drug ribavirin. Given the molecular overlap between medulloblastoma biology and known ribavirin activity, the authors investigated the preclinical efficacy of repurposing ribavirin as a targeted therapeutic in cell and animal models of medulloblastoma.

METHODS Multiple in vitro assays were performed using human ONS-76 (a primitive SHH model) and D425 (an aggressive group 3 model) cells. The impacts of ribavirin on cellular growth, death, migration, and invasion were quantified using proliferation and Cell Counting Kit-8 (CCK-8) assays, flow cytometry with annexin V (AnnV) staining, scratch wound assays, and Matrigel invasion chambers, respectively. Survival following daily ribavirin treatment $(100 \mathrm{mg} / \mathrm{kg}) \mathrm{was}$ assessed in vivo in immunodeficient mice intracranially implanted with D425 cells.

RESULTS Compared to controls, ribavirin treatment led to a significant reduction in medulloblastoma cell growth (ONS-76 proliferation assay, $p=0.0001$; D425 CCK-8 assay, $p<0.0001$ ) and a significant increase in cell death (flow cytometry for AnnV, ONS-76, $p=0.0010$; D425, $p=0.0284$ ). In ONS-76 cells, compared to controls, ribavirin significantly decreased cell migration and invasion (Matrigel invasion chamber assay, $p=0.0012$ ). In vivo, ribavirin significantly extended survival in an aggressive group 3 medulloblastoma mouse model compared to vehicle-treated controls $(p=$ 0.0004).

CONCLUSIONS The authors demonstrate that ribavirin, a clinically used drug known to inhibit elF4E and EZH2, has significant antitumor effects in multiple preclinical models of medulloblastoma, including an aggressive group 3 animal model. Ribavirin may represent a promising targeted therapeutic in medulloblastoma.

https://thejns.org/doi/abs/10.3171/2020.8.PEDS20561

KEYWORDS ribavirin; medulloblastoma; brain tumor; cancer; repurposing; targeted therapy; oncology

$\mathrm{M}$ EDUlLoblastoma is an embryonal tumor of the cerebellum that is among the most common pediatric brain tumors. ${ }^{1}$ Its malignant disease process with a propensity for leptomeningeal ${ }^{2}$ and hematogenous $^{3}$ spread necessitates the use of aggressive multimodal treatment. Standard therapy includes maximal safe resection; multiagent chemotherapy with vincristine, cisplatin, cyclophosphamide, and/or lomustine; and radiation of the entire craniospinal axis. ${ }^{4}$ These surgical and adjuvant ap- proaches lead to significant neurotoxicity in the developing nervous system, with patients subsequently experiencing severe cognitive, endocrine, and psychosocial deficits. ${ }^{1}$ Consequently, of surviving patients, many experience persistent tumor- and treatment-related sequelae that substantially affect quality of life. ${ }^{5}$ There is an urgent clinical need for more tailored therapeutics that target underlying disease processes while also reducing the doses of standard therapies necessary to achieve clinical benefit.

ABBREVIATIONS AnnV = annexin V; CCK-8 = Cell Counting Kit-8; elF4E = eukaryotic initiation factor 4E; EZH2 = enhancer of zeste homolog 2; SHH = Sonic Hedgehog. SUBMITTED June 26, 2020. ACCEPTED August 24, 2020.

INCLUDE WHEN CITING Published online February 5, 2021; DOI: 10.3171/2020.8.PEDS20561.

${ }^{*}$ N.S. and B.M.T. contributed equally to this work. 
Medulloblastoma is subdivided into four distinct molecular subgroups: Wnt, Sonic Hedgehog (SHH), group 3 (Myc driven), and group $4 .{ }^{6}$ Advances in our molecular understanding of medulloblastoma have elucidated groupspecific alterations in key proteins involved in transcription and translation in native and cancer biology. These include Myc, a family of proto-oncogenes with critical roles in proliferating cells, ${ }^{7}$ eukaryotic initiation factor $4 \mathrm{E}$ (eIF4E), a rate-limiting determinant of translation; ${ }^{8}$ and enhancer of zeste homolog 2 (EZH2), a histone methyltransferase deemed a master regulator of transcription. ${ }^{9-11}$ In SHH medulloblastoma, N-Myc and eIF4E have been implicated as central mediators of multiple oncogenic processes. ${ }^{12-15} \mathrm{~N}-\mathrm{Myc}$ has also been closely linked to EZH2, ${ }^{16,17}$ which has roles in SHH medulloblastoma viability, proliferation, and self-renewal. ${ }^{18}$ Group 3 medulloblastoma is closely linked with c-Myc, whose direct relationship with eIF4E has been well characterized in multiple cancers. ${ }^{19,20}$ Perhaps unsurprisingly, the eIF4E family of proteins has been implicated in group 3 medulloblastoma signaling and response to treatment. ${ }^{21} \mathrm{EZH} 2$ has similarly emerged as an important protein in group 3 medulloblastoma and a potentially promising target for therapy in this aggressive subgroup..$^{10,22,23}$

EZH2 and eIF4E have been successfully targeted in clinical trials in other cancers, but to our knowledge, they have not been widely applied to the clinical management of medulloblastoma. Both of these proteins are known to be modulated by ribavirin (structural name $1-\beta-\mathrm{D}-$ ribofuranosyl-1,2,4-triazole-3-carboxamide, formula $\left.\mathrm{C}_{8}-\mathrm{H}_{12}-\mathrm{N}_{4}-\mathrm{O}_{5}\right),{ }^{24}$ an antiviral drug approved by the FDA that has recently been repurposed for possible therapy in multiple cancers ${ }^{24}$ including glioblastoma, ${ }^{25}$ atypical teratoid/rhabdoid tumor, ${ }^{26}$ nasopharyngeal carcinoma, ${ }^{27}$ leukemia, ${ }^{28,29}$ breast cancer, ${ }^{30}$ and more. Given the molecular overlap between medulloblastoma biology and ribavirin activity, as well as the well-documented safety profile of ribavirin in humans, we investigated the preclinical efficacy of repurposing ribavirin as a targeted therapeutic in cell and animal models of medulloblastoma.

\section{Methods}

\section{Medulloblastoma Models and Cell Culture}

Human medulloblastoma cell lines ONS-76 (SHH driven, adherent in culture) and D425 (group 3, grown as neurospheres) were obtained from the Japanese Cancer Research Resources Bank and the Eberhart laboratory at Johns Hopkins University, respectively. ONS-76 and D425 cells were cultured in RPMI (Lonza) and DMEM (Gibco), respectively. All media were supplemented with $10 \%$ fetal bovine serum (Lonza) and $1 \%$ penicillin/streptomycin. Cells were maintained at $37^{\circ} \mathrm{C}$ in $5 \% \mathrm{CO}_{2}$-humidified incubators. All cells were authenticated and tested for mycoplasma infection.

For in vitro experiments, cells were treated with varied doses of ribavirin (Sigma-Aldrich) or ultrapure $\mathrm{H}_{2} \mathrm{O}$ (Invitrogen) as vehicle control.

\section{Cell Growth/Proliferation Assay}

ONS-76 cells $\left(2.5 \times 10^{4}\right)$ were seeded in 6-well plates.
After adhering overnight, cells were treated with daily ribavirin or ultrapure $\mathrm{H}_{2} \mathrm{O}$. Cells were collected and counted using a Malassez slide (Invitrogen) 2, 4, and 6 days following treatment initiation.

\section{Cell Counting Kit-8 Assay}

D425 cells $\left(2.0 \times 10^{3}\right)$ were seeded in 96-well plates and subsequently treated with daily ribavirin or ultrapure $\mathrm{H}_{2} \mathrm{O}$. Cell viability was quantified using Cell Counting Kit-8 (CCK-8; Dojindo) and a microplate reader (PerkinElmer) per the manufacturers' instructions.

\section{Flow Cytometry}

ONS-76 cells $\left(2.5 \times 10^{4}\right)$ and D425 cells $\left(5.0 \times 10^{4}\right)$ were seeded in 6-well plates and treated with daily ribavirin or ultrapure $\mathrm{H}_{2} \mathrm{O}$ for 6 and 7 days, respectively. Cells were then collected and prepared for flow cytometry with staining for annexin V (AnnV; product A35110, ThermoFisher Scientific). AnnV positivity was assessed using a BD FACS Celesta flow cytometer (BD Biosciences, Becton, Dickinson and Company) and analyzed using FlowJo software (FlowJo LLC).

\section{Scratch Wound/Migration Assay}

ONS-76 cells $\left(2.5 \times 10^{4}\right)$ were seeded in 6-well plates and grown until they formed a confluent monolayer. Following treatment with ribavirin or $\mathrm{H}_{2} \mathrm{O}$, a scratch wound was made in the center of the well by scraping it with a P200 pipette tip. Wells were then washed with phosphatebuffered saline (Invitrogen) and incubated to allow cells to migrate into the space cleared by the scratch. Cell migration was assessed by capturing images from identical locations along each wound using a Zeiss Observer Z.1 AX10 microscope at 0 and 12 hours after the scratch.

\section{Invasion Assay}

Invasion assays were performed using Corning BioCoat Matrigel Invasion Chambers. ONS-76 cells $\left(1.0 \times 10^{5}\right)$ were pretreated with ribavirin or $\mathrm{H}_{2} \mathrm{O}$ and plated in the top chamber in serum-free medium. Cells were allowed to invade into the lower chamber (containing culture medium with $10 \%$ fetal bovine serum) for 24 hours. The bottom of each insert was then fixed and stained with crystal violet. The numbers of invading cells were assessed using a Zeiss Observer Z.1 AX10 microscope.

\section{Intracranial Xenograft Model}

Athymic immunodeficient mice were obtained from Charles River Laboratories and housed in the Johns Hopkins University animal facility with free access to food and water under an Animal Care and Use Committee-approved protocol. An intracranial model of D425 was established as described previously. ${ }^{26}$ Briefly, following intraperitoneal injection of pharmaceutical grade anesthesia and analgesia, a burr hole was drilled in the left parietal bone of each study animal. D425 cells $\left(1.25 \times 10^{5}\right)$ were implanted intracranially in 26 mice by using Hamilton syringes (Hamilton Company). Animals received a daily intraperitoneal injection $(200 \mu \mathrm{l})$ of ribavirin $(100 \mathrm{mg} / \mathrm{kg})$ 
A ONS-76 Cell Growth/Proliferation

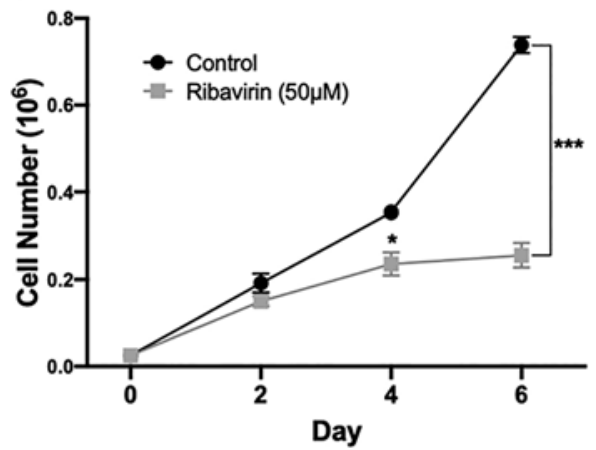

B D425 Cell Growth/Proliferation

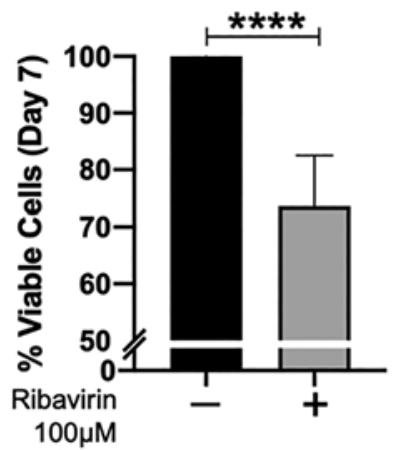

FIG. 1. Ribavirin inhibits medulloblastoma cell growth. A: Cell growth/proliferation assay performed with ONS-76 cells demonstrates a decreased cell number in the presence of ribavirin (black curve, vehicle $\left[\mathrm{H}_{2} \mathrm{O}\right]$ control; gray curve, ribavirin $\left.50 \mu \mathrm{M}\right)$. B: CCK-8 assay performed with D425 cells demonstrates a decreased cell number in the presence of ribavirin $(100 \mu \mathrm{M})\left({ }^{*} p<\right.$ $0.05,{ }^{* * *} p<0.001,{ }^{* * * *} p<0.0001$, ribavirin vs control, $n=3$ ).

(Johns Hopkins Hospital) or ultrapure $\mathrm{H}_{2} \mathrm{O}$ (Invitrogen) as control.

\section{Statistical Analysis}

Data for in vitro experiments represent at least three biological repeats. Differences between groups were assessed using t-tests. Survival analysis was performed using Kaplan-Meier curves and log-rank tests. Statistics were performed using GraphPad Prism 8.4 software, and $\mathrm{p}$ values $<0.05$ were considered statistically significant.

\section{Results}

\section{Ribavirin Inhibits Cell Growth and Induces Cell Death in Medulloblastoma}

We first tested the effects of ribavirin on cell growth and viability. A cell growth/proliferation assay was performed with the adherent ONS-76 cell line, and ribavirin treatment demonstrated significant inhibition of cell growth following 4 (control 354,200 vs ribavirin 235,800 cells, $\mathrm{p}=0.0200$ ) and 6 (control 738,500 vs ribavirin 255,800 cells, $p=0.0001$ ) days of treatment (Fig. 1A). We then performed a CCK-8 assay with nonadherent D425 cells and observed a significant reduction in cell growth following 7 days of treatment (control $100 \%$ vs ribavirin $74 \%$ viable cells, $\mathrm{p}<0.0001$, data normalized to controls) (Fig. 1B).

To understand whether these reductions in cell growth occurred due to induction of cell death, we next used flow cytometry with staining for AnnV. This analysis demonstrated that ribavirin led to a significant increase in the proportion of AnnV-positive cells (i.e., dying cells) in both ONS-76 (control 3.4\% vs ribavirin 14.1\%, $\mathrm{p}=0.0010$ ) and $\mathrm{D} 425$ (control $4.3 \%$ vs ribavirin $10.3 \%, \mathrm{p}=0.0284$ ) cells (Fig. 2).

Taken together, our data suggest that ribavirin inhibits medulloblastoma cell growth by inducing cell death.

\section{Ribavirin Reduces Medulloblastoma Cell Migration and Invasion}

We next investigated ribavirin's effects on medulloblastoma cell migration through scratch wound assays with

ONS-76 cells. We were unable to perform this assay with D425 cells due to their nonadherent nature. Representative photographs demonstrated a reduction in ONS-76 cell migration into the center of the scratch wound 12 hours after the scratch was performed (Fig. 3A).

To simulate invasion through a basement membrane, we then performed invasion assays with Matrigel-coated inserts and demonstrated that ribavirin treatment led to a significant reduction in the invasive capacity of ONS-76 cells (Fig. 3B). In total, our findings demonstrate that ribavirin may provide an effective means of inhibiting medulloblastoma cell migration and invasion.

\section{Ribavirin Extends Survival in a Group 3 Medulloblastoma Animal Model}

Last, we investigated the efficacy of ribavirin as monotherapy in an aggressive group 3 medulloblastoma model established via intracranial injection of D425 cells (Fig. 4A). Animals received daily $100 \mathrm{mg} / \mathrm{kg}$ intraperitoneal injections of pharmaceutical grade ribavirin or vehicle control based on its known safety and efficacy in prior studies

A

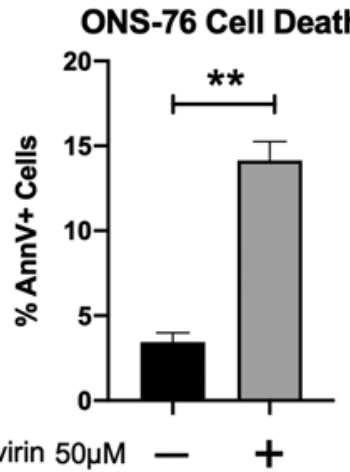

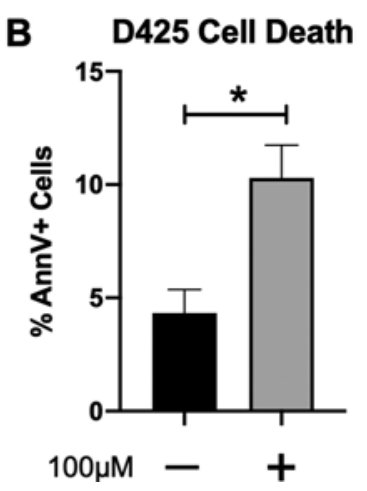

B D425 Cell Death

cllow

FIG. 2. Ribavirin promotes medulloblastoma cell death. Flow cytometrybased quantification of AnnV-positive cells (dead cells) following ribavirin treatment in ONS-76 (A) and D425 (B) cells. Ribavirin treatment significantly increased cell death in both cell lines tested $\left({ }^{*} p<0.05,{ }^{* *} p<0.01\right.$, ribavirin vs vehicle $\left[\mathrm{H}_{2} \mathrm{O}\right]$ control, $n=3$ ). 
A

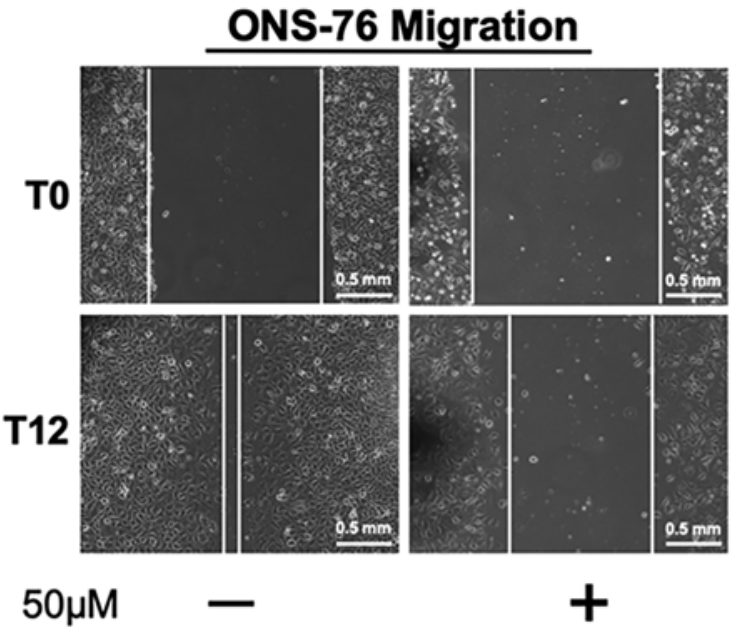

B ONS-76 Invasion

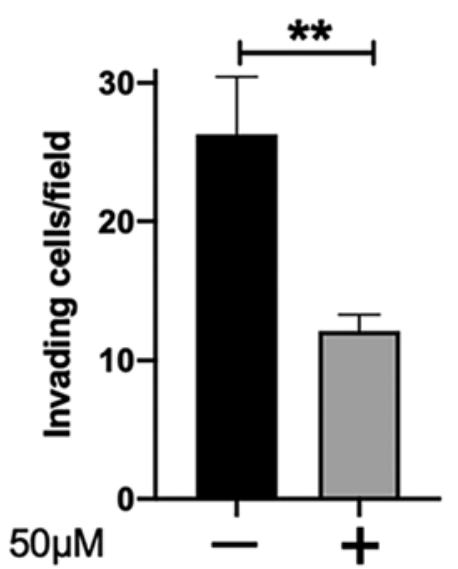

FIG. 3. Ribavirin reduces medulloblastoma cell migration and invasion. A: Migration of ONS-76 cells treated with vehicle $\left(\mathrm{H}_{2} \mathrm{O}\right)$ control or ribavirin $(50 \mu \mathrm{M})$ was assessed using the scratch wound assay. Representative photographs were taken immediately after the scratch (T0) and 12 hours later (T12). Bar $=0.5 \mathrm{~mm}$. B: Invasion of ONS-76 cells treated with vehicle or ribavirin $(50 \mu \mathrm{M})$ was assessed using inserts coated with Matrigel matrix. Ribavirin treatment significantly decreased the number of cells invading through the Matrigel matrix $\left({ }^{* *} p<0.01\right.$, ribavirin vs vehicle $\left[\mathrm{H}_{2} \mathrm{O}\right]$ control, $n=3$ ).

and maximally tolerated dose experiments. ${ }^{25-27}$ Ribavirin treatment led to a significant extension in median survival (control 25 days vs ribavirin 29 days, $\mathrm{p}=0.0004)$ (Fig. 4B).

\section{Discussion}

\section{Study Rationale}

Current treatment paradigms for medulloblastoma frequently result in poor clinical outcomes due to the underlying disease (i.e., tumor recurrence and metastasis) as well as severe treatment-related toxicities that markedly reduce quality of life..$^{1,4,5}$ There is a clinical need for tailored treatments that leverage knowledge of medulloblastoma cancer biology and work in concert with existing therapies that are clinically useful but limited by CNS toxicity. Two proteins with demonstrated group-specific alterations in medulloblastoma include eIF4E, a rate-limiting component of translation, and EZH2, a histone methyltransferase with broad epigenetic control of native and cancer biology. ${ }^{6-18}$ eIF4E and EZH2 have both been modulated by the FDA-approved antiviral drug ribavirin in multiple malignancies, including pediatric and adult brain tumors. ${ }^{24-26}$ With this molecular rationale, we undertook the present laboratory investigation of the novel use of ribavirin as a therapeutic for medulloblastoma.

\section{Key Results and Interpretation}

We first showed that ribavirin decreased cell growth and increased cell death in ONS-76 (SHH) and D425 (group 3) medulloblastoma models in vitro. ONS-76 cells were more sensitive to ribavirin treatment, with efficacy demonstrated at lower doses than those used in D425 cells (50 vs $100 \mu \mathrm{M}$, respectively). These effects are consistent with prior mechanistic studies; Mainwaring and Kenney previously demonstrated that eIF4E is a target of $\mathrm{SHH}$ signaling and also required for the proliferation of medulloblastoma precursor cells. ${ }^{15}$ Abdelfattah and colleagues additionally showed in group 3 medulloblastoma models that silencing of eIF4E led to a reduction in cell viability, growth, and colony formation. ${ }^{21}$ Multiple studies have demonstrated that pharmacologic ${ }^{18}$ and gene silencing ${ }^{31}$ methods of inhibiting EZH2 reduced proliferation and increased apoptosis in SHH medulloblastoma cells. Zhang and colleagues similarly demonstrated in group 3 medulloblastoma that pharmacologic inhibition of EZH2 inhibited cell growth and induced cell cycle arrest. ${ }^{10}$

We next demonstrated that ribavirin reduced medulloblastoma cell migration and invasion. These findings may be of particular clinical relevance, given medulloblastoma's unique propensity for metastasis. ${ }^{1-3}$ While the exact mechanism in medulloblastoma is unknown, we speculate based on work in other cancers that these effects may be multifactorial and facilitated in part by targeting of two factors: matrix metalloproteinases, a family of endopeptidases involved in extracellular matrix degradation and epithelial-to-mesenchymal transition, and thrombospondin-1, a matricellular protein with an essential role in Myc-induced migration and invasion in medulloblastoma. ${ }^{24,25,32-35}$ Both matrix metalloproteinases and thrombospondin-1 are known to be associated with eIF4E and EZH 2. ${ }^{32,36-38}$ Further study is warranted to understand the exact mechanisms of action involved in ribavirin-mediated repression of migration and invasion.

Finally, we found that daily ribavirin treatment led to a significant increase in survival in a group 3 medulloblastoma model in vivo. This finding was likely driven by mechanistic underpinnings similar to those discussed above and is consistent with prior work demonstrating reduced medulloblastoma tumor growth in vivo through targeting of eIF4E and EZH2. ${ }^{18,21}$ Ultimately, our results are consistent with the documented roles of eIF4E and EZH2 in medulloblastoma and the known ability of ribavirin to modulate these two proteins.

In addition to aligning with medulloblastoma cancer 
A

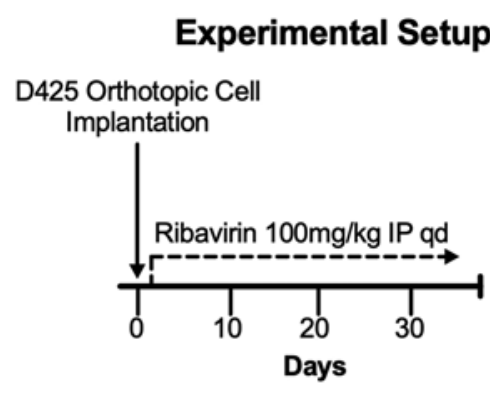

B

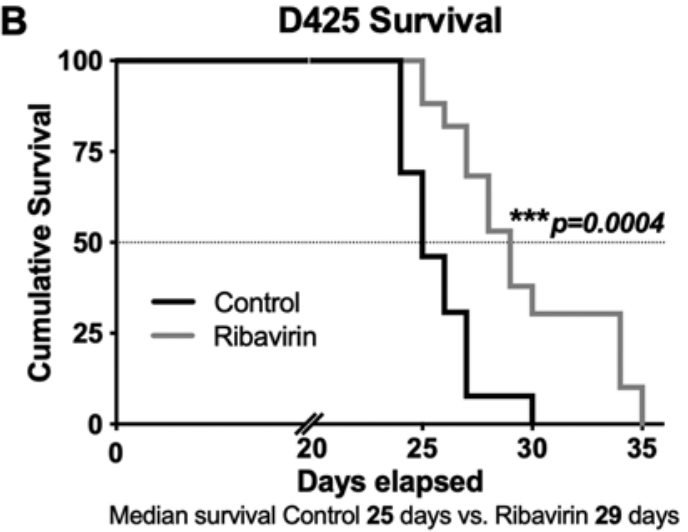

FIG. 4. Ribavirin increases survival in an orthotopic xenograft model of group 3 medulloblastoma. A: Experimental setup. Athymic immunodeficient ( $\mathrm{Nu} / \mathrm{Nu}$ ) mice were intracranially implanted with D425 cells and treated daily with an intraperitoneal injection of ribavirin $100 \mathrm{mg} / \mathrm{kg}$ or vehicle $\left(\mathrm{H}_{2} \mathrm{O}\right)$ control. B: Kaplan-Meier survival analysis for in vivo experiment. Ribavirin treatment led to a statistically significant increase in median survival compared to vehicle-treated controls ( 29 vs 25 days, respectively; $p=0.0004$, log-rank test, $n=13$ per group).

biology, our results are also consistent with prior studies using ribavirin in other cancers. In a preclinical study in atypical teratoid rhabdoid tumor, an aggressive pediatric brain tumor with loss of integrase interactor 1 (with consequent increase in EZH2), ribavirin similarly decreased cell proliferation, viability, migration, and invasion in vitro and increased survival in vivo. ${ }^{21}$ These effects were shown to be mediated by a reduction in EZH2 and phosphoeIF4E protein expression. Similar work and mechanistic underpinnings have been demonstrated in glioblastoma ${ }^{25}$ nasopharyngeal carcinoma, leukemia, ${ }^{28,29}$ breast cancer, ${ }^{30}$ and more.

\section{Clinical Relevance}

Ribavirin may be of particular clinical interest due to the evidence base already supporting its safe and effective clinical use in pediatric and adult populations. It crosses the blood-brain and blood-CSF barriers, ${ }^{25,26,39-41}$ and dosing profiles are well studied. Adult patients receiving treatment for hepatitis $\mathrm{C}$ previously received doses of $800-1200 \mathrm{mg} / \mathrm{day}$, and clinical trials in cancer patients have shown safe administration of $2800 \mathrm{mg} /$ day without toxicity. ${ }^{29,42}$ In a study using ribavirin for the treatment of subacute sclerosing panencephalitis in a pediatric population, patients tolerated doses as high as $30 \mathrm{mg} / \mathrm{kg}$ three times per day. ${ }^{41}$ Our dose of $100 \mathrm{mg} / \mathrm{kg}$ in mice equates to $12 \mathrm{mg} / \mathrm{kg}$ for a $20-\mathrm{kg}$ human child based on common dose conversion calculations..$^{43}$ Our preclinical in vitro and in vivo experiments therefore demonstrated efficacy using ribavirin doses that are well tolerated and below clinically achievable doses. ${ }^{43}$ The main clinical toxicity of ribavirin is hemolytic anemia, of which we did not observe any signs or symptoms in our study.

An especially appealing feature of ribavirin is its documented ability to safely sensitize to mainstays of treatment for medulloblastoma, including radiation, ${ }^{25,27}$ vincristine, ${ }^{21}$ cisplatin ${ }^{44}$ and other cytotoxic agents. ${ }^{29}$ Prior work suggests that these radio- and chemosensitizing features are mediated in part by inhibition of eIF4E and EZH2. ${ }^{24}$
Clinically, ribavirin has also been safely used in combination therapy regimens and has been shown to lower the effective doses of other chemotherapeutics, including a 1000 -fold reduction in the effective dose of cytarabine in acute myeloid leukemia. ${ }^{29}$ Further study is needed to determine optimal combination therapy regimens and timing in a cancer-specific manner. Nevertheless, there is a strong rationale and need for additional preclinical studies investigating the use of ribavirin in combination therapy regimens for medulloblastoma.

\section{Drug Repurposing}

Drug repurposing represents a pragmatic and efficient alternative to traditional drug development pipelines that can be prohibitively expensive and time consuming. This approach instead allows for faster development with lower safety risks and economic burden. ${ }^{45}$ It may also allow for improved access to novel therapeutics in lowerresource settings. To this end, perhaps the most appealing aspect of repurposing ribavirin as an anticancer agent is that it is already approved by the FDA and has been studied in clinical trials for multiple neoplastic indications, including acute myeloid leukemia, ${ }^{28}$ oropharyngeal squamous cell carcinoma (NCT01721525), metastatic breast cancer (NCT01056757), hepatocellular carcinoma (NCT00375661, NCT00834860, NCT02771405), lymphoma (NCT02717949, NCT03585725), and prostate cancer (UMIN000012521, UMIN000021107). This clinical use of ribavirin, coupled with our preclinical rationale and study, provide a preliminary basis for future preclinical and clinical investigation of ribavirin as a repurposed therapeutic for medulloblastoma.

\section{Study Limitations}

We recognize several limitations of our study. We investigated ribavirin as monotherapy in ONS-76 and D425 models of medulloblastoma. If used clinically, ribavirin would be used as one component of a broader multimodal 
treatment regimen; ribavirin should therefore be investigated preclinically in combination therapy regimens with current treatment modalities (i.e., radiation, cisplatin, and vincristine). This work should ideally include evaluation in patient-derived xenograft models. While there is a strong molecular rationale for the use of ribavirin in medulloblastoma, the precise mechanism of action underlying our experimental findings is not characterized in the present work and should be evaluated in future studies. This work should include analysis of molecular targeting as well as cell cycle analysis in order to inform treatment planning and combination therapy regimens. We also note that, while ribavirin has demonstrated preclinical efficacy in multiple cancers and performed well in early-phase clinical trials, there is currently a lack of robust phase 3 clinical trial data describing efficacy of ribavirin in cancer. Future preclinical and clinical work is needed to determine the full potential of ribavirin as a repurposed therapeutic in medulloblastoma and other cancers.

\section{Conclusions}

There is a clinical need for tailored treatments that leverage knowledge of medulloblastoma cancer biology and improve the toxicity profile of existing therapies. We investigated the preclinical efficacy of ribavirin as a repurposed and targeted therapeutic in medulloblastoma based on the known roles of eIF4E and EZH2 in medulloblastoma and the documented ability of ribavirin to modulate these proteins in multiple cancers. We demonstrate that ribavirin represents a potentially promising medulloblastoma therapeutic due to its efficacy in multiple in vitro assays and survival benefit in an aggressive animal model of medulloblastoma. Ribavirin may be an appealing candidate for further study in medulloblastoma given its existing regulatory approval, multitargeted activity, and ability to sensitize to radiation and chemotherapy in multiple cancers. Our work provides a foundation for further preclinical and clinical evaluation of ribavirin as a targeted therapeutic in medulloblastoma.

\section{Acknowledgments}

We thank Dr. Charles G. Eberhart for generously providing the D425 cell line. We additionally thank the Hunterian Neurosurgical Research Laboratory for helpful comments and editing.

\section{References}

1. Northcott PA, Robinson GW, Kratz CP, et al. Medulloblastoma. Nat Rev Dis Primers. 2019;5(1):11.

2. Groves MD. New strategies in the management of leptomeningeal metastases. Arch Neurol. 2010;67(3):305-312.

3. Garzia L, Kijima N, Morrissy AS, et al. A hematogenous route for medulloblastoma leptomeningeal metastases. Cell. 2018;172(5):1050-1062.e14.

4. Packer RJ, Gajjar A, Vezina G, et al. Phase III study of craniospinal radiation therapy followed by adjuvant chemotherapy for newly diagnosed average-risk medulloblastoma. $J$ Clin Oncol. 2006;24(25):4202-4208.

5. Veneroni L, Boschetti L, Barretta F, et al. Quality of life in long-term survivors treated for metastatic medulloblastoma with a hyperfractionated accelerated radiotherapy (HART) strategy. Childs Nerv Syst. 2017;33(11):1969-1976.
6. Northcott PA, Korshunov A, Witt H, et al. Medulloblastoma comprises four distinct molecular variants. J Clin Oncol. 2011;29(11):1408-1414.

7. Roussel MF, Robinson GW. Role of MYC in medulloblastoma. Cold Spring Harb Perspect Med. 2013;3(11):a014308.

8. Siddiqui N, Sonenberg N. Signalling to eIF4E in cancer. Biochem Soc Trans. 2015;43(5):763-772.

9. Kim KH, Roberts CW. Targeting EZH2 in cancer. Nat Med. 2016;22(2):128-134.

10. Zhang $\mathrm{H}$, Zhu $\mathrm{D}$, Zhang Z, et al. EZH2 targeting reduces medulloblastoma growth through epigenetic reactivation of the BAI1/p53 tumor suppressor pathway. Oncogene. 2020; 39(5):1041-1048.

11. Northcott PA, Buchhalter I, Morrissy AS, et al. The wholegenome landscape of medulloblastoma subtypes. Nature. 2017;547(7663):311-317.

12. Beltran $\mathrm{H}$. The $\mathrm{N}$-myc oncogene: maximizing its targets, regulation, and therapeutic potential. Mol Cancer Res. 2014; 12(6):815-822.

13. Wu J, Tang Q, Yang L, et al. Interplay of DNA methyltransferase 1 and EZH2 through inactivation of Stat 3 contributes to $\beta$-elemene-inhibited growth of nasopharyngeal carcinoma cells. Sci Rep. 2017;7(1):509.

14. Charron F. Linking Hedgehog, translation, and mTORC1 in medulloblastoma. Dev Cell. 2017;43(6):655-656.

15. Mainwaring LA, Kenney AM. Divergent functions for eIF4E and S6 kinase by sonic hedgehog mitogenic signaling in the developing cerebellum. Oncogene. 2011;30(15):1784-1797.

16. Corvetta D, Chayka O, Gherardi S, et al. Physical interaction between MYCN oncogene and polycomb repressive complex 2 (PRC2) in neuroblastoma: functional and therapeutic implications. J Biol Chem. 2013;288(12):8332-8341.

17. Dardenne E, Beltran H, Benelli M, et al. N-myc induces an EZH2-mediated transcriptional program driving neuroendocrine prostate cancer. Cancer Cell. 2016;30(4):563-577.

18. Miele E, Valente S, Alfano V, et al. The histone methyltransferase EZH2 as a druggable target in SHH medulloblastoma cancer stem cells. Oncotarget. 2017;8(40):68557-68570.

19. Jones RM, Branda J, Johnston KA, et al. An essential E box in the promoter of the gene encoding the mRNA cap-binding protein (eukaryotic initiation factor 4E) is a target for activation by c-myc. Mol Cell Biol. 1996;16(9):4754-4764.

20. Lin C-J, Malina A, Pelletier J. c-Myc and eIF4F constitute a feedforward loop that regulates cell growth: implications for anticancer therapy. Cancer Res. 2009;69(19):7491-7494.

21. Abdelfattah N, Rajamanickam S, Panneerdoss S, et al. MiR584-5p potentiates vincristine and radiation response by inducing spindle defects and DNA damage in medulloblastoma. Nat Commun. 2018;9(1):4541.

22. Vo BT, Li C, Morgan MA, et al. Inactivation of Ezh2 upregulates Gfil and drives aggressive Myc-driven group 3 medulloblastoma. Cell Rep. 2017;18(12):2907-2917.

23. Smits M, van Rijn S, Hulleman E, et al. EZH2-regulated DAB2IP is a medulloblastoma tumor suppressor and a positive marker for survival. Clin Cancer Res. 2012;18(15):40484058.

24. Casaos J, Gorelick NL, Huq S, et al. The use of ribavirin as an anticancer therapeutic: will it go viral? Mol Cancer Ther. 2019;18(7):1185-1194.

25. Volpin F, Casaos J, Sesen J, et al. Use of an anti-viral drug, ribavirin, as an anti-glioblastoma therapeutic. Oncogene. 2017;36(21):3037-3047.

26. Casaos J, Huq S, Lott T, et al. Ribavirin as a potential therapeutic for atypical teratoid/rhabdoid tumors. Oncotarget. 2018;9(8):8054-8067.

27. Huq S, Casaos J, Serra R, et al. Repurposing the FDAapproved antiviral drug ribavirin as targeted therapy for nasopharyngeal carcinoma. Mol Cancer Ther. 2020;19(9): 1797-1808. 
28. Assouline S, Culjkovic B, Cocolakis E, et al. Molecular targeting of the oncogene eIF4E in acute myeloid leukemia (AML): a proof-of-principle clinical trial with ribavirin. Blood. 2009;114(2):257-260.

29. Assouline S, Culjkovic-Kraljacic B, Bergeron J, et al. A phase I trial of ribavirin and low-dose cytarabine for the treatment of relapsed and refractory acute myeloid leukemia with elevated eIF4E. Haematologica. 2015;100(1):e7-e9.

30. Pettersson F, Yau C, Dobocan MC, et al. Ribavirin treatment effects on breast cancers overexpressing eIF4E, a biomarker with prognostic specificity for luminal B-type breast cancer. Clin Cancer Res. 2011;17(9):2874-2884.

31. Alimova I, Venkataraman S, Harris P, et al. Targeting the enhancer of zeste homologue 2 in medulloblastoma. Int $J$ Cancer. 2012;131(8):1800-1809.

32. Pettersson F, Del Rincon SV, Emond A, et al. Genetic and pharmacologic inhibition of eIF4E reduces breast cancer cell migration, invasion, and metastasis. Cancer Res. 2015;75(6): $1102-1112$.

33. Gkogkas CG, Khoutorsky A, Cao R, et al. Pharmacogenetic inhibition of eIF4E-dependent Mmp9 mRNA translation reverses fragile X syndrome-like phenotypes. Cell Rep. 2014; 9(5):1742-1755.

34. Robichaud N, del Rincon SV, Huor B, et al. Phosphorylation of eIF4E promotes EMT and metastasis via translational control of SNAIL and MMP-3. Oncogene. 2015;34(16):20322042.

35. Zhou L, Picard D, Ra YS, et al. Silencing of thrombospondin-1 is critical for Myc-induced metastatic phenotypes in medulloblastoma. Cancer Res. 2010;70(20):8199-8210.

36. Shin YJ, Kim JH. The role of EZH2 in the regulation of the activity of matrix metalloproteinases in prostate cancer cells. PLoS One. 2012;7(1):e30393.

37. Decarlo L, Mestel C, Barcellos-Hoff M-H, Schneider RJ. Eukaryotic translation initiation factor $4 \mathrm{E}$ is a feed-forward translational coactivator of transforming growth factor $\beta$ early protransforming events in breast epithelial cells. Mol Cell Biol. 2015;35(15):2597-2609.

38. Zhang Y, Zheng D, Zhou T, et al. Androgen deprivation promotes neuroendocrine differentiation and angiogenesis through CREB-EZH2-TSP1 pathway in prostate cancers. Nat Commun. 2018;9(1):4080.

39. Colombo G, Lorenzini L, Zironi E, et al. Brain distribution of ribavirin after intranasal administration. Antiviral Res. 2011; 92(3):408-414.

40. Gilbert BE, Wyde PR, Wilson SZ, Robins RK. Aerosol and intraperitoneal administration of ribavirin and ribavirin triacetate: pharmacokinetics and protection of mice against intracerebral infection with influenza A/WSN virus. Antimicrob Agents Chemother. 1991;35(7):1448-1453.

41. Hosoya M, Shigeta S, Mori S, et al. High-dose intravenous ribavirin therapy for subacute sclerosing panencephalitis. Antimicrob Agents Chemother. 2001;45(3):943-945.
42. Abenavoli L, Mazza M, Almasio PL. The optimal dose of ribavirin for chronic hepatitis $\mathrm{C}$ : from literature evidence to clinical practice: the optimal dose of ribavirin for chronic hepatitis C. Hepat Mon. 2011;11(4):240-246.

43. Reagan-Shaw S, Nihal M, Ahmad N. Dose translation from animal to human studies revisited. FASEB J. 2008;22(3): 659-661.

44. Jin J, Xiang W, Wu S, et al. Targeting eIF4E signaling with ribavirin as a sensitizing strategy for ovarian cancer. Biochem Biophys Res Commun . 2019;510(4):580-586.

45. Bertolini F, Sukhatme VP, Bouche G. Drug repurposing in oncology-patient and health systems opportunities. Nat Rev Clin Oncol. 2015;12(12):732-742.

\section{Disclosures}

Dr. Brem reports receiving research funding that includes grants from NIH, Johns Hopkins University, Arbor Pharmaceuticals, Bristol-Myers Squibb, AcuityBio Corp., ${ }^{*}$ and philanthropy and being a consultant for AsclepiX Therapeutics, StemGen, InSightec, Accelerating Combination Therapies,* Camden Partners, ${ }^{*}$ LikeMinds, Inc., * Galen Robotics, Inc., * NexImmune, and Nurami Medical.* Ms. Tyler reports being a patent holder of Accelerating Combination Therapies.* (*Includes equity or options.)

\section{Author Contributions}

Conception and design: Tyler, Huq, Kannapadi, Casaos, Skuli. Acquisition of data: Tyler, Huq, Kannapadi, Casaos, Lott, Felder, Serra, Gorelick, Ruiz-Cardozo, Ding, Cecia, Medikonda, Ehresman. Analysis and interpretation of data: Tyler, Huq, Casaos, Serra, Skuli. Drafting the article: Tyler, Huq. Critically revising the article: all authors. Reviewed submitted version of manuscript: all authors. Approved the final version of the manuscript on behalf of all authors: Tyler. Statistical analysis: Tyler, Huq, Skuli. Administrative/technical/material support: Brem. Study supervision: Brem, Skuli.

\section{Supplemental Information}

Previous Presentations

Portions of this study were presented in electronic poster form at the AANS 2020 Annual Scientific Meeting (virtual) and CNS 2020 Annual Meeting (virtual).

\section{Correspondence}

Betty M. Tyler: Johns Hopkins University School of Medicine, Baltimore, MD.btyler@jhmi.edu. 